\title{
Pathomorphological features of some organs in dirofilariasis of dogs
}

\author{
Tatiana Alymova ${ }^{1, *}$, Nikolay Krasnoslobodtsev ${ }^{2}$, and Evgeniy Shapiro ${ }^{3}$ \\ 1“Kobets" IE, Veterinary Center "Beethoven”, Khabarovsk, Russia \\ 2"DM Plus" LLC, "Druzhok" Veterinary Clinic, Khabarovsk, Russia \\ ${ }^{3}$ Far Eastern State Agrarian University, Blagoveshchensk, Russia
}

\begin{abstract}
Dirofilariasis (from Lat. "diro, filum" meaning "evil thread") is a parasitic disease caused by helminths of Dirofilaria. Filamentous helminths are of the white color and belong to the class of nematodes. On the territory of the Far East, there are 2 types of dirofilarias: D. immitis and D. repens. This parasitic infection is characterized by a very long course; a worm-infested individual can be a carrier of worms for up to three years. At the same time, they poison the body with dangerous products of metabolism and cause numerous pathological changes. Dirofilarias (most of them) are located in the heart and lungs. Microfilarias regularly circulate in the blood of animals. Examining the organs of dogs which have suffered from dirofilariasis, it can be concluded that the presence of helminths in the blood of animals causes damage to the entire body of the dog, injuring every organ.
\end{abstract}

\section{Introduction}

Parasitic infestation is one of the main problems of the animal world. Parasites, living inside the body of a pet, not only affect internal organs, the circulatory system, but also disrupt the usual metabolism. As a result, some internal micro-processes do not work correctly.

The problem is that most owners can't correctly detect a parasitic infestation. Unfortunately, the presence of helminths in the feces is not always the primary sign. At the initial stage the dog's behavior is absolutely standard and the symptoms are not observed. One of the main errors of primary diagnostics is that helminths must be in the feces, and, allegedly, if they are not there, the animal is completely healthy.

Parasites can live not only in the intestinal cavity, but also in the heart, lungs, stomach walls, and other important organs. At the primary stage, it is difficult to detect the disease. That is why the owners go to the doctor with a disease of a neglected form. Parasites living in the walls of the organ can penetrate into the soft tissues, disrupting its work.

In dirofilariosis, the mosquito bites a carnivorous animal and during blood-sucking it swallows blood along with microfilariae. During 8-14 days in the body of a mosquito, the larvae shed twice and reach an invasive form. They migrate from the stomach of

'Corresponding author: t.alymova2015@yandex.ru 
mosquitoes to the malpighian vessels where their metamorphosis takes place. Next, the larvae penetrate the mosquito's mouth, tears the palpi and lips, and during repeated blood sucking the microfilaria enters the bloodstream of the definitive host. Larvae migrate to the site of localization by hematogenic and lymphogenic ways. Worms become mature in 6-9 months, and their life expectancy is 2-3 years old.

In one milliliter of blood, there can be found up to 10 thousand of microdirofilariae.

There are several stages of D. Immitis dirofilariasis development:

Stage 1. Asymptomatic or mild course. There are no clinical signs and changes on radiography, no changes in laboratory parameters. There can be a change in the habit, load intolerance, and periodic cough.

Stage 2. A moderate course. There is periodic cough, slight or moderate load intolerance, slight loss of condition, increased lung noise (crepitation), weak or moderate changes on the X-ray - an increase in the right atrium. Anemia, proteinuria.

Stage 3. Severe course. There is anemia, weight loss, exercise intolerance, frequent breathing at rest, severe and persistent cough, shortness of breath, fainting, sneezing with blood or bloody mucus, pleurisy or ascites. The X-ray shows an enlarged right ventricle and an enlarged pulmonary artery, and a change in the lungs. Anemia, thrombocytopenia, and proteinuria.

Stage 4. Vena cava syndrome. An emergency medical condition. There is sudden collapse, hemoglobinuria, and shortness of breath. It is often fatal without surgical intervention.

Dirofilaria immitis cause dominant pathological changes in the heart, the lungs, sometimes in the kidneys.

After a certain time given for reproduction and development of larvae for about 3-5 months, there appear pathological changes. The first changes are found in the terminal arteries of the lobes of the lungs. There are inflammatory and morphological changes caused by mature microfilariae, namely proliferation and thickening of vascular intima and media, vacuolization, cellular infiltration and subendothelial edema. The expanded alveoli are filled with eosinophils and macrophages. Constant exposure to helminths causes thrombotic changes in the pulmonary arteries, and granulomatous foci of inflammation turn into a chronic form and there is an accumulation of fibrin in these places. Then the logical result is an overload of the small circle of blood circulation, which causes heart failure with hypertrophy and dilation of the right parts of the heart of the pulmonary arteries. Due to venous hyperemia, liver congestion and fibrosis occur, which can contribute to ascites or peritonitis. Due to the work of immune cells in the animal's body, local changes in the kidneys are observed, namely, the glomerules are disrupted. This contributes to the appearance of proteinuria, amyloidosis and interstitial nephritis. It is also possible to localize dirofilariae and their larvae in the central nervous system.

The main purpose of this work is to study the pathomorphological features of organs in dogs with dirofilariasis. The main tasks are:

1) to determine pathomorphological changes in the heart in dirofilariasis of dogs;

2) to determine pathomorphological changes in the lungs in dirofilariasis of dogs;

3) to study the histomorphological features of the kidneys in dirofilariasis of dogs.

\section{Materials and Methods}

The material for the study was 8 dogs aged from 3 to 9 years old.

The animals lived in the conditions of the city of Khabarovsk with their owners. The clinical examination was performed using the standard method. The condition of the animal was assessed visually; the auscultation of the heart was performed. When detecting heart rhythm disorders, there was performed x-ray examination (at the GENORAY OSCAR 
classic device), ultrasound diagnostics (at MINDRAY RESONA 7 ultrasound device), express test for microfilarias (at "Canine Heartworm Ag (CHW Ag)" by Hospitex Diagnostics, Italy), and also there were made native blood smears which were then examined under a microscope (Macromed-1. 2-LED). The heart, kidneys, lungs, helminths, arteries and jugular vein were studied after the death of a pet. The selected material was left in a $10 \%$ aqueous solution of neutral formalin. Pathomorphological research was carried out in the laboratory of pathomorphology of the Far Eastern State Agrarian University.

\section{Results}

At the autopsy of dead dogs, the most pronounced changes were found in the cardiovascular system, lungs and kidneys.

Dirofilarias were most often found in the right half of the heart where they caused a violation of the integrity of the endothelial lining. Helminths were attached to the front end, forming hemorrhages, erosions and ulcers. In places of erosion, the deposition of fibrin filaments with the formation of sclerotic masses was observed. The valvular heart apparatus responded very actively to their presence, as well as the endothelial lining; as a result, the valves were injured and endocarditis was formed. The valves were assembled in veil-like structures that cannot completely cover the passage between the ventricle and the atrium. Therefore, the blood with dirofilarias and microfilarias filled the cavity of the ventricles.

The heart had a rounded oval shape with well-defined atrial tension. On the coronary transverse there was no fat accumulation, in some cases, there was mucosal atrophy of the fat that had been before. The top of the heart was obtuse; in some cases it was bifurcated. The pericardial wall was thickened and grayish-white in color, and the visceral layer of the heart joint was in the same state. It had a flaccid consistency and a light brownish color (Fig. 1).

In the bronchi and lungs, there were observed limited areas of pulmonary fibrosis with the formation of linear cords, as well as changes in the pulmonary parenchyma, namely the presence of infiltrates.

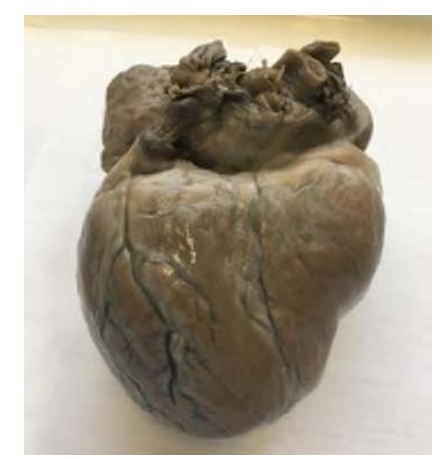

Fig. 1. The picture shows the heart of a dog, a breed of Central Asian shepherd, 5 years old.

On the section, the muscles are flaccid with a rusty brown hue, the heart cavities are expanded and filled with loose blood clots which contain different numbers of dirofilarias and microfilarias; the thickness of the right ventricle is 5-7 times greater than the one in the left one (Fig.2). 


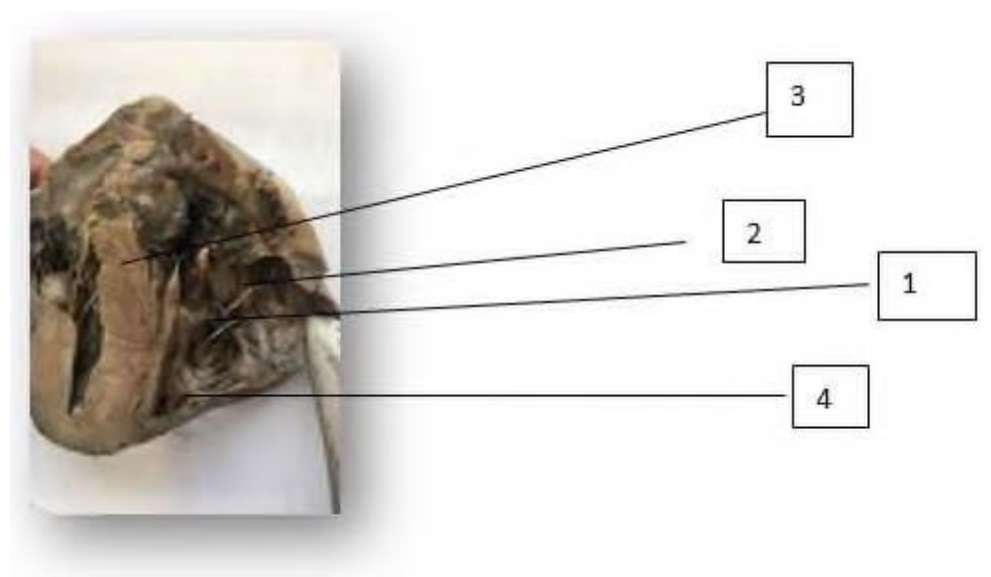

Fig. 2. The heart of the dog in the section. 1 - dirofilarias in the right ventricle, 2 - endocarditis, 3 hypertrophied areas of the heart, 4 - areas of hemorrhage.

In the bronchi and lungs there were observed limited areas of pulmonary fibrosis with the formation of linear cords, as well as changes in the pulmonary parenchyma, namely the presence of infiltrates.

The lungs were found to be full of venous blood and edema. There was also alveolar and focal emphysema with a predominant lesion of the apical lobes, serous-catarrhal pneumonia and serous-fibrinous pleurisy (Fig. 3).

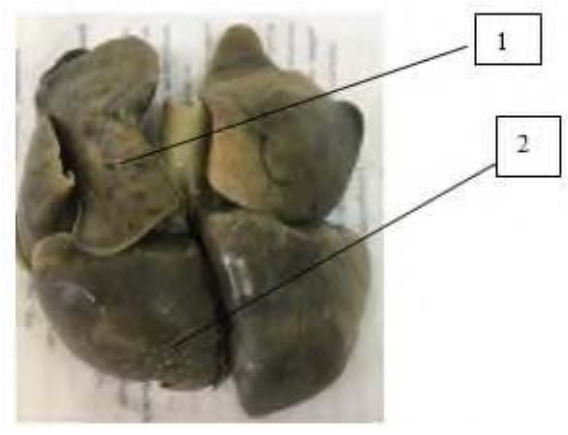

Fig. 3. The lungs of the dog (6 years old). 1 - alveolar emphysema; 2 - abscess sites.

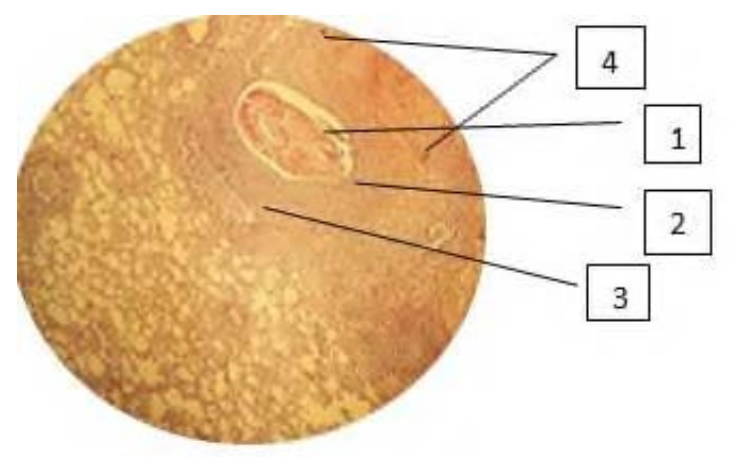

Fig 4. Cross section of the dog's lungs (8 years old). 
Increase of approx. x 18 vol. x4. Stained with hematoxylin and eosin

1 - microfilaria; 2 - distelectase; 3 - sclerosis with lymphoid infiltration; 4 - focal hemorrhages.

In the study of the kidneys there was observed serous-fibrinous effusion in the cavity of the glomerular capsule in the form of homogeneous eosinophilic masses; glomeruli and tubules in hollow areas are atrophic and replaced by connective hyalinosis tissue; the glomeruli are saved and hypertrophied in the extruded sections; a capsule of ShumlyanskyBowman is thickened; capillary loops are scleroid; tubules are dilated with flattened epithelium and focal granular cytoplasm; arteries are scleroid and vitrified; stroma is with areas of sclerosis and lymphohistiocytic infiltration.

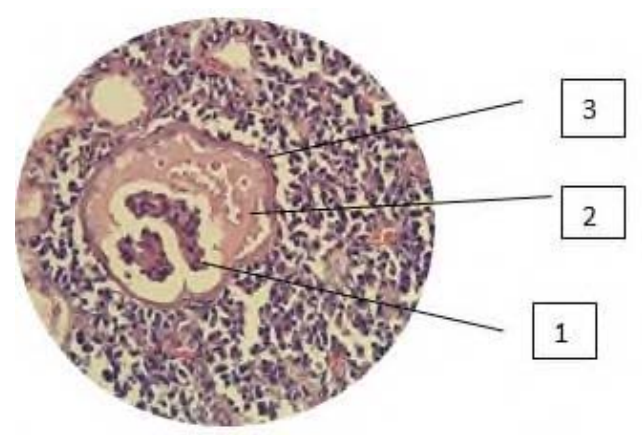

Fig. 5. The glomerulus of the kidney of the dog with dirofilariasis.

Increase of approx. x 18 vol. x4. Stained with hematoxylin and eosin. 1 - a small amount in mesangial cells; 2 - homogeneous eosinophilic mass; 3 - thickened Shumlyansky- Bowman capsule

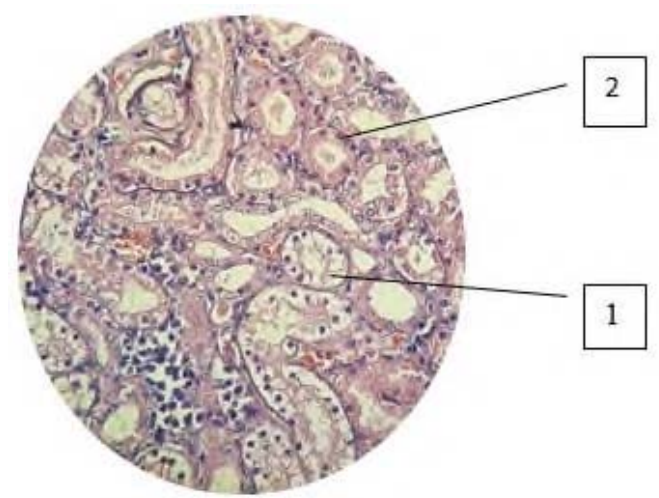

Fig. 6. Tubules of the kidney.

Increase of approx. $x 18$ vol. x4. Stained with hematoxylin and eosin.

1- hydropic dystrophy of the epithelium of the proximal tubules; 2-granular dystrophy.

\section{Discussion}

These studies of the cardiovascular system are consistent with the research of such scientists as Shinkorenko A. N., Kolesnikov P. V., Karaulov V. V. These scientists describe that hypertrophic cardiomyopathy occurs as a result of dirofilariasis (Fig. 2), which is 
characterized by thickening of the heart muscle. The myocardium may be thickened uniformly or in any of the departments predominantly. [1]

\section{Conclusions}

At autopsy of dead animals it is possible to note the following changes in the body of animals:

1. In the cardiovascular system there are pronounced erosive and ulcerative endocarditis, endocardial fibroelastosis, parietal and valvular endocarditis.

2. In the lungs there is pneumonia of various genesis;

3. In the kidneys there is exudative extracapillary glomerulonephritis and granular dystrophy.

\section{References}

1. A. N. Shinkarenko, P. V. Kolesnikov Cardio-pulmonological pathology in dirofilariasis of dogs, Russian Parasitological Journal 4 (2009)

2. H. Mirahmadi, A. Maleki, R. Hasanzadeh Ocular dirofilariasis by Dirofilaria immitis in a child in Iran: A case report and review of the literature. Parasitol Int. 66(1), 978-981. (2017); doi:10.1016/j.parint.2016.10.022

3. F. Dantas-Torres, RP Lia, Barbuto M, et al. Ocular dirofilariosis by Dirofilaria immitis in a dog: first case report from Europe, J Small Anim Pract. 50(12), 667-669. (2009) doi:10.1111/j.1748-5827.2009.00846.x

4. G. Gioia, L. Lecová . Highly sensitive multiplex PCR for simultaneous detection and discrimination of Dirofilaria immitis and Dirofilaria repens in canine peripheral blood. Vet Parasitol 172(1-2), 160-163 (2010) doi:10.1016/j.vetpar.2010.04.027

5. D. Tahir, F. Bittar Molecular survey of Dirofilaria immitis and Dirofilaria repens by new real-time TaqMan ${ }^{\circledR}$ PCR assay in dogs and mosquitoes (Diptera: Culicidae) in Corsica (France). Vet Parasitol. 235, 1 -7 (2017);. doi:10.1016/j.vetpar.2017.01.002

6. M.S. Latrofa, F. Montarsi . Molecular xenomonitoring of Dirofilaria immitis and Dirofilaria repens in mosquitoes from north-eastern Italy by real-time PCR coupled with melting curve analysis. Parasit Vectors. 5, 76. (2012) doi:10.1186/1756-3305-576

7. C.A. FerreiraFirst molecular identification of mosquito vectors of Dirofilaria immitis in continental Portugal. Parasit Vectors 8, 139. (2015) doi:10.1186/s13071-015-0760-2

8. G. Capelli. Risk of canine and human exposure to Dirofilaria immitis infected mosquitoes in endemic areas of Italy. Parasit Vectors, 6, 60. (2013) doi:10.1186/17563305-6-60

9. D. Vezzani Dirofilariasis in Argentina: historical review and first report of Dirofilaria immitis in a natural mosquito population. Vet Parasitol, 136(3-4), 259-273. (2006) doi:10.1016/j.vetpar.2005.10.026

10. H.P. Fuehrer Dirofilaria in Humans, Dogs, and Vectors in Austria (1978-2014)-From Imported Pathogens to the Endemicity of Dirofilaria repens. PLoS Negl Trop Dis. 10(5), e0004547. Published (2016)

11. G. Capelli Recent advances on Dirofilaria repens in dogs and humans in Europe. Parasit Vectors. 11(1), 663. Published (2018) doi:10.1186/s13071-018-3205-x 
12. C. Genchi Subcutaneous dirofilariosis (Dirofilaria repens), an infection spreading throughout the old world. Parasit Vectors.;10(Suppl 2), 517. (2017) doi:10.1186/s13071-017-2434-8

13. C. Silaghi Development of Dirofilaria immitis and Dirofilaria repens in Aedes japonicus and Aedes geniculatus. Parasit Vectors. 10(1), 94. (2017) doi:10.1186/s13071-017-2015-x

14. V. Velev Human Dirofilariasis in Bulgaria Between 2009 and 2018. Helminthologia. 56(3), 247-251. (2019) doi:10.2478/helm-2019-0016

15. A. Diakou Dirofilaria infections in dogs in different areas of Greece. Parasit Vectors. 9(1), 508. (2016) doi:10.1186/s13071-016-1797-6

16. V. Sabūnas Dirofilaria repens in dogs and humans in Lithuania. Parasit Vectors. 12(1), 177.(2019). doi:10.1186/s13071-019-3406-y 